\title{
Analysis of a plasma test cell including non-neutrality and complex collision mechanisms
}

\author{
Paul N. Giuliano* and Iain D. Boyd ${ }^{\dagger}$ \\ University of Michigan, Department of Aerospace Engineering, Ann Arbor, MI 48103, USA
}

\begin{abstract}
This study concerns the validation of numerical models commonly used in simulations of Hall effect and other plasma thrusters and their plumes. A comparison is made of ion beam simulations with experimental data from a simplified plasma test cell in order to better characterize an environment representative of such electric propulsion devices. The simulation utilized is the DSMC-PIC tool MPIC representing heavy species as macro-particles in a non-quasineutral method. The addition and refinement of a more realistic electric potential solver in MPIC leads to the ability to simulate the effects of electrically biased surfaces in the test cell data. In addition, an analysis of secondary electron production via heavy species interactions is carried out due to the appearance of a higher-pressure phenomenon likely related to electron physics.
\end{abstract}

\section{Nomenclature}

$\phi \quad$ Electric potential, $\mathrm{V}$

$T_{a} \quad$ Temperature of species $a, \mathrm{~K}$ or eV

$n_{a} \quad$ Number density of species $a, \mathrm{~m}^{-3}$

$P \quad$ Pressure, Torr

$\sigma \quad$ Collision cross-section, $\mathrm{m}^{2}$

Ex Collision energy, eV

$m_{a} \quad$ Mass of species $a, \mathrm{~kg}$

$k_{B} \quad$ Boltzmann's constant, $\mathrm{J} / \mathrm{K}$

$I \quad$ Current, A

$V \quad$ Applied bias voltage, $\mathrm{V}$

$\lambda \quad$ Collision mean free path, $\mathrm{m}$

$\nu \quad$ Collision frequency, $\mathrm{s}^{-1}$

Subscript

e Electron

$i \quad$ Ion

$r \quad$ Reference quantity

\section{Introduction}

This study concerns an electric propulsion modeling effort with a focus on stepping back from fulldevice Hall effect thruster (HET) modeling in order to develop and validate tools which can simulate and predict the fundamental physical processes occurring in HET operation. There is a particular interest in the simulation of plasma transport mechanisms through the use of kinetic methods such as direct simulation Monte Carlo (DSMC) and particle-in-cell (PIC) algorithms. Kinetic methods have the advantage of not limiting electron behavior to fluid assumptions, such as having a Maxwellian distribution, which have proven

*Ph.D. Candidate, Department of Aerospace Engineering, Ann Arbor, MI 48109, AIAA Member.

${ }^{\dagger}$ James E. Knott Professor, Department of Aerospace Engineering, Ann Arbor, MI 48109, AIAA Fellow. 
to be grossly inaccurate due to the prevalence of nonequilibrium phenomena such as inelastic processes and sheath formation.

However, it is generally understood that important transport mechanisms, such as anomalous electron transport, are collective effects arising in magnetized, low-temperature devices such as HETs, requiring high fidelity simulation of more than just electron physics. The inelastic processes and transport mechanisms of the heavy species involved in these devices must be understood so that an anomalous transport model can be built incrementally, introducing the complexities of multiple species, device geometry, and magnetic fields.

The aim of this computational study is to mirror the developments of an experimental counterpart in which a high-voltage xenon ion beam is accelerated into a controlled test cell for the purpose of observing heavy-species collision mechanisms. This study utilizes the kinetic simulation tool MPIC to compare simulated ion beam predictions to measured values via a comparison of current collection at specified electrodes while taking into account the biased potential of the test cell, the non-neutrality of the injected ion beam, and the projected influence of secondarily created electrons via particle-wall and particle-particle ionization.

Section II of this study describes the numerical domain, tools, models, and experimental data used in our analysis, including the development of a new electric potential solver in MPIC. Section III shows the results of current collection comparisons between MPIC and experimental data. Lastly, Section IV describes a basic analysis in which a possible source of secondary electrons is discussed.

\section{Method}

\section{A. Numerical domain}

The experimental setup which is the focus of this simulation study is previously described by Wirz, et al., ${ }^{1}$ located at UCLA and consists of a $1500 \mathrm{~V}$ xenon ion beam being accelerated into a small, cylindrical facility held at a controlled pressure and temperature. The facility is simulated numerically using a simplified axisymmetric domain, seen in Figure 1. The domain, termed "the test cell", is $152 \mathrm{~mm}$ long and $48.24 \mathrm{~mm}$ in diameter with an inlet used for particle injection, an outlet for particle exit, and a line of rotation along the axis.

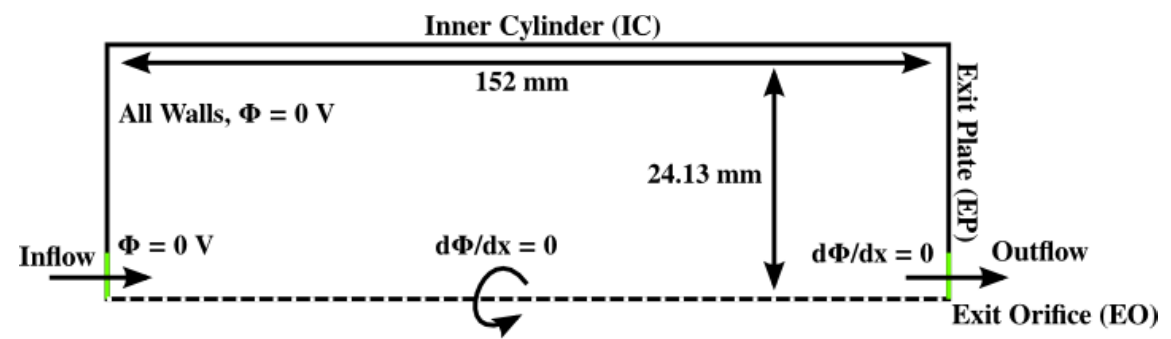

Figure 1. Numerical domain of the Wirz experiment, detailing inlet, outlet, and symmetry conditions as well as voltage conditions at the walls.

As noted in the Figure, the two regions of particular interest are the inner cylinder (IC) and the exit plate (EP), representing electrically isolated surfaces on which current is measured in the UCLA experiments. In addition, recent measurements have included the current exiting the domain through the outlet as the Exit Orifice (EO). The currents collected on the IC, EP, and EO provide datasets that will be compared to the simulations. All walls and the inlet are assigned a Dirichlet boundary condition using a voltage of $0 \mathrm{~V}$ for the typical unbiased test case. The axis of rotation and outlet are defined by a Neumann boundary condition using a zero potential gradient. This numerical domain is held constant for all simulations except for the bias voltage at the IC which is biased positively and negatively to mirror experimental investigations.

The measured data are organized into three sets: DS1, the unbiased test cell data, DS4, the biased lowercurrent test cell data, and DS5, the biased higher-current test cell data. "Unbiased" and "biased" refers to the application of a voltage to the IC in certain operating conditions which is explained in further detail in the Results. Each data set has a unique amount of total current within the test cell, previously recorded experimentally and then simplified using a curve-fit in order to be adapted as numerical inputs. The details of the operating pressures, total currents, and current curve-fits for DS1, DS4, and DS5 are provided in Tables 2 and 3 in the Appendix. 


\section{B. Numerical tools}

The simulation tool used in this study is MPIC, ${ }^{2}$ an axi-symmetric particle-based direct simulation Monte Carlo (DSMC) code capable of simulating nonequilibrium, rarefied flows with a PIC algorithm allowing for the calculation of electrostatic forces. The PIC module determines the charge density at the nodes in the mesh based on the proximity of each particle to the surrounding nodes. The charge density is then used to compute the electric field at each node. This can be accomplished either by forcing quasineutrality via the Boltzmann relation or a detailed electron-fluid model, or in a non-quasineutral way using a recently implemented direct Poisson's equation solver which uses the charge densities directly. The potential is then differentiated spatially to obtain the electric fields. Subsequently, acceleration due to the electric field affects the trajectory of heavy charged species. A typical test cell simulation is first populated with neutral particles until the correct background pressure is achieved after which the beam ions are injected. The simulation is allowed to reach a steady state after which time-averaged solutions are generated. A typical ion beam simulation is made up of about 300,000 to 1,000,000 particles, depending on operating conditions, with a domain of about 4,000 cells. A timestep of $3 \times 10^{-8} \mathrm{~s}$ is used for a typical run of 600,000 timesteps which takes between 6 to 10 hours of wall time.

\section{Electric potential solver}

In order to correctly simulate the biased walls of the test cell as well as increase the simulation physics of MPIC, the electric potential solver is advanced from a quasineutral Boltzmann relation assumption to a direct solution of Poisson's equation derived from the difference in charge density of ions and electrons. In the present study, only ions are considered.

The original model used in MPIC simulations is that of the Boltzmann relation, one of the most widely used electron models in plasma simulation. The Boltzmann relation is a solution of the electron momentum equation derived using some nontrivial assumptions: the fluid electron flow is isothermal, electron pressure obeys the ideal gas law, and magnetic fields can be neglected.

In the model, electrons are assumed to have constant temperature. As a result, the pressure gradient and electric forces in the electron momentum equation are balanced and the potential, $\phi$, at any point is then:

$$
\phi=\phi_{r}+T_{e} \ln \left(\frac{n_{e}}{n_{r}}\right)
$$

where $\phi_{r}$ and $n_{r}$ are reference electric potential and density, respectively, and are chosen to match the input conditions of the domain which, in this case, are the potential and density of the ion beam at the injection point of the test cell. While the density of the ion beam changes based on operating condition, the reference potential is set to 0 Volts at the inlet. In addition, $T_{e}$ is the electron temperature in $\mathrm{eV}$, and $n_{e}$ is the electron number density. For these simulations, $T_{e}$ is set to the injection temperature of the ion beam, $296 \mathrm{~K}$, and $n_{e}$ is set to ion number density, $n_{i}$, throughout the simulation using the assumption of quasineutrality.

This model is inherently inappropriate for the ion beam and test cell because quasineutrality is not valid for the ion-only injection. In addition, the Boltzmann relation is unable to recognize electric potential boundary conditions within the domain, instead solely using the reference potential. An important aspect of the test cell is the ability to vary the voltage on the IC in order to observe ion collection and, eventually, secondary electron-collection.

The development of a more physics-based electron simulation approach is described by Boyd and Yim ${ }^{3}$ who modeled the electrons as a fluid using conservation equations of mass, momentum, and energy. Each equation is assumed to describe the electron fluid at steady state and is subsequently solved for specific fundamental electron properties: electron velocity, plasma potential, and electron temperature, respectively. This transformation also results in a set of Poisson-like equations with source terms. The model was implemented into MPIC by $\mathrm{Cai}^{4}$ via a finite-element solver.

However, for the purpose of this study, this "detailed electron model" is inappropriate for the test cell as it also assumes quasineutrality. Therefore, the implementation of the Poisson-like equation solver has been modified to instead solve the Poisson equation directly from ion charge density without any quasineutral assumptions about the electrons. The Poisson equation can be represented in cylindrical coordinates as

$$
\nabla^{2} \phi=\frac{1}{r} \frac{\delta}{\delta r}\left[r \frac{\delta}{\delta r} \phi(r, z)\right]+\frac{\delta^{2}}{\delta z^{2}} \phi(r, z)=\frac{q}{\epsilon_{0}}\left(n_{i}-n_{e}\right) .
$$


This non-neutral method has the ability to use all electric potential boundary conditions as well as add the future possibility to include electrons in a non-quasineutral way, such as through their secondary production due to energetic particle collisions with walls.
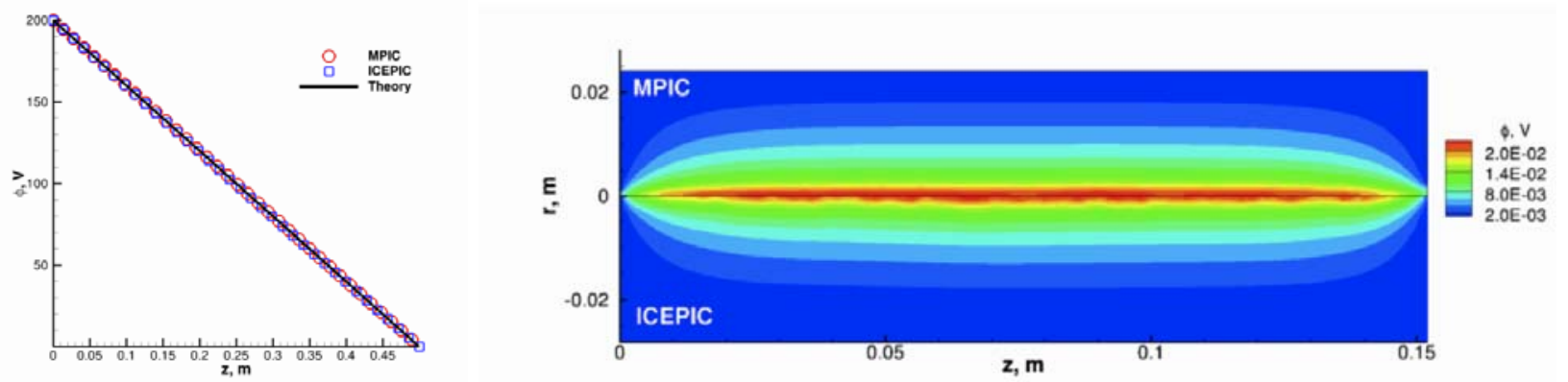

Figure 2. Results of the 1d Laplace's equation validation test (left) and the 2d collisionless Poisson's equation test (right) showing good correlation between MPIC and ICEPIC/theory.

The Poisson solver is first validated using an exact solution of a one-dimensional Laplace's equation alongside another code, ICEPIC, which also uses a direct Poisson solve. ICEPIC is the Improved Concurrent Electromagnetic Particle-in-Cell simulation toolkit which is a fully kinetic, 1d/2d/3d, Cartesian/cylindrical PIC simulation tool and a good benchmark for Poisson solver accuracy. The Laplace equation takes the form of the Poisson equation in the presence of zero charge density and can be easily solved, i.e.,

$$
\nabla^{2} \phi=0, \phi=A x+B,
$$

making for an ideal, simple test case to ensure that the finite-element solving method is in working order. Using a 1-d domain with $\phi=200 \mathrm{~V}$ at $x=0$ and $0 \mathrm{~V}$ at $x=L$, the coefficients are found to be $B=200 \mathrm{~V}$ and $A=200 \mathrm{~V} / L$. The results of the test case plotting MPIC, ICEPIC, and the exact solution can be seen in Figure 2 where the MPIC Poisson solver matches exactly the solutions of ICEPIC and theory.

Next, the finite-element Poisson solver is tested in two dimensions in a domain similar to that of the ion beam and test cell for a more realistic test case. The domain follows exactly the parameters outlined in the Numerical Domain section but with a collisionless ion beam corresponding to a low-pressure operating condition beam current. This test case is carried out in both MPIC and ICEPIC in order to compare electric potential contours resulting from this simple ion beam throughout the domain. The results of the MPIC/ICEPIC test case can be seen in Figure 2. The electric potential contours show a good match except for at the inlet and outlet regions where MPIC uses a Neumann boundary condition and ICEPIC uses a Dirichlet condition.

\section{Results}

\section{A. Unbiased test cell}

A full comparison between MPIC and UCLA measured results is first compiled for the unbiased data set, DS1, in order to provide a baseline current collection comparison for the simplest case. In addition, the unbiased test case allows for a standard comparison of the Poisson equation solver with the original Boltzmann relation solver wherein biased-voltage boundary conditions are of no concern. A comparison of current collection for the three electrodes and the total current can be seen in Figures 3-4.

In Figure 3, the MPIC IC current collection matches well with the measurements while the computed EP consistently lies below the measured data. In the case of the EP current, there is the possibility of unresolved beam divergence in the simulated ion beam which would lead to off-axis ion injection and more EP current being collected than simply through scattering. It can be seen that the Poisson solver matches very well with the Boltzmann relation method, proving the efficacy of both methods for this unbiased environment. Figure 4 shows the EO and total current collection comparisons with MPIC agreeing very well with the measurements. 

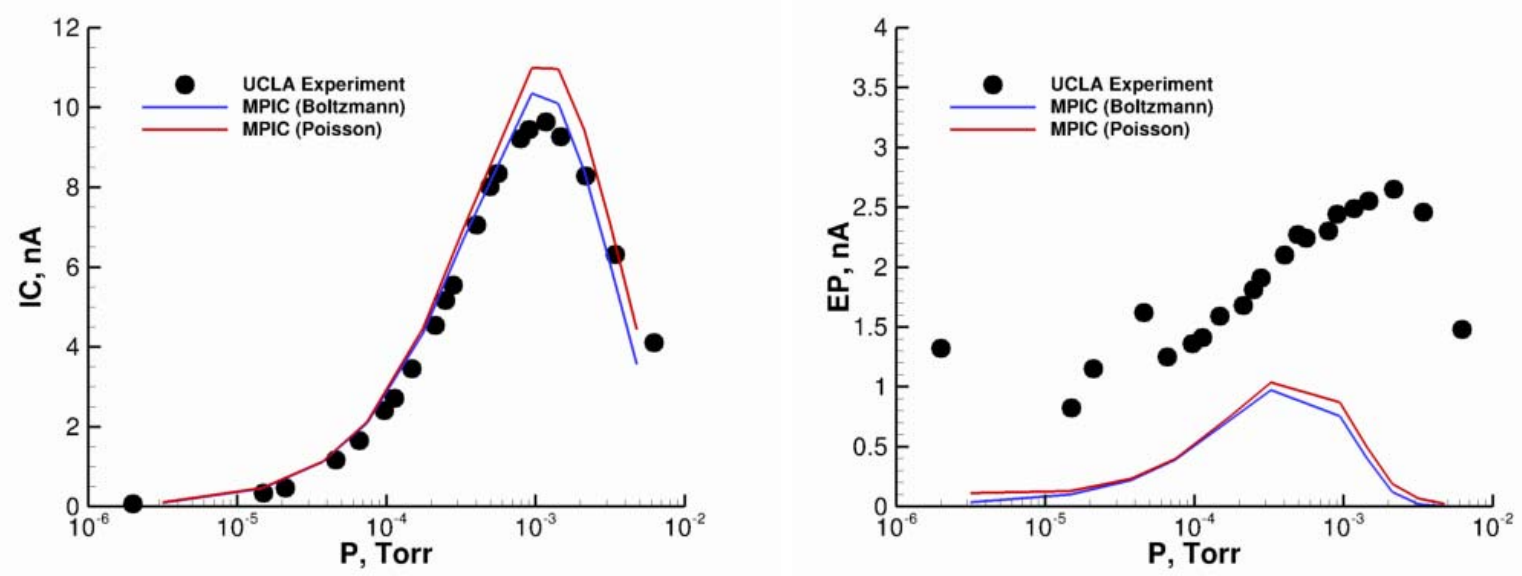

Figure 3. Current collection at the IC (left) and EP (right) for the unbiased data set (DS1) versus operating condition pressure for MPIC and UCLA measurements.
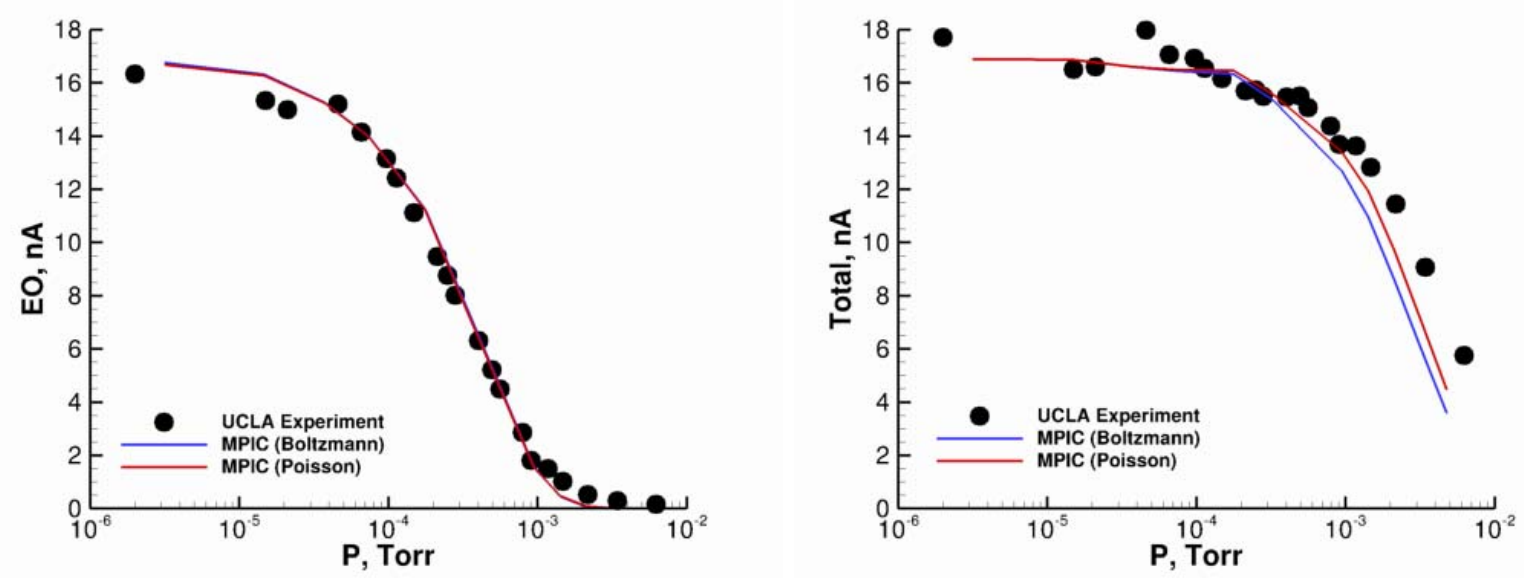

Figure 4. Current collection at the EO (left) and total domain (right) for the unbiased data set (DS1) versus operating condition pressure for MPIC and UCLA measurements. 

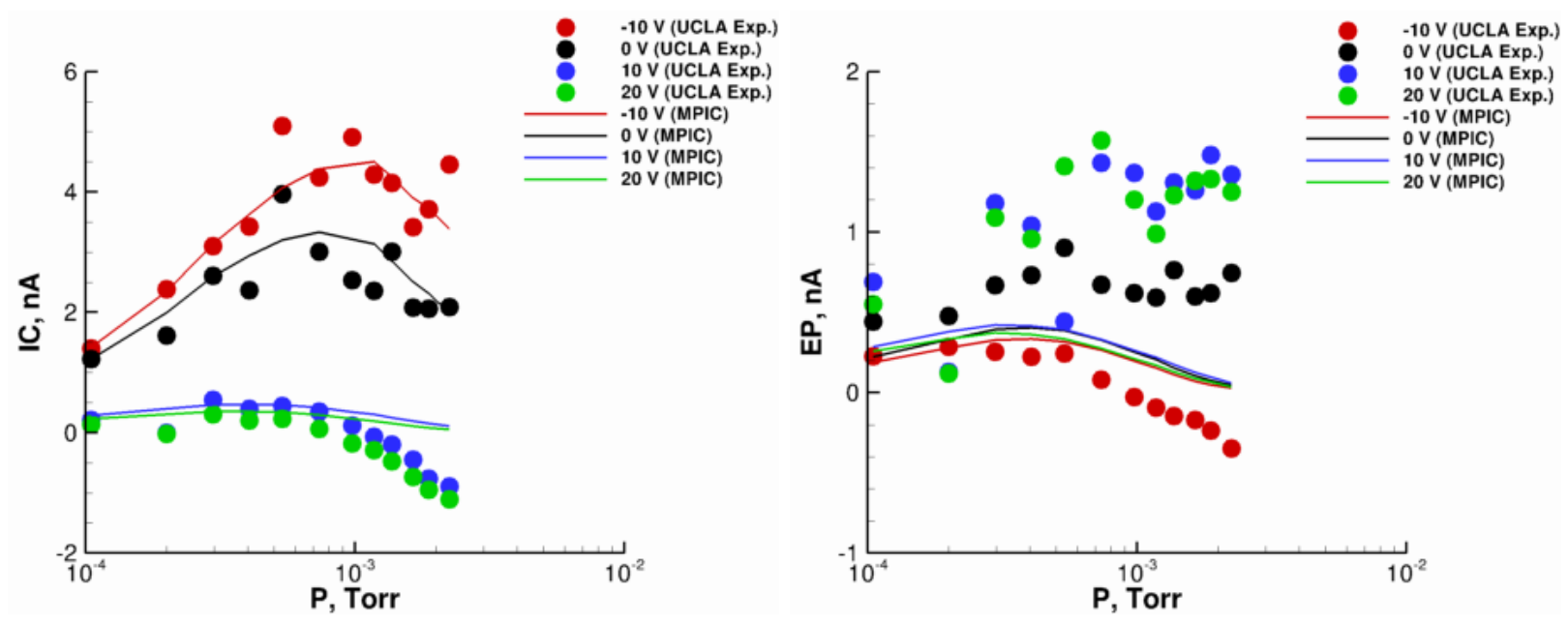

Figure 5. Current collection at the IC (left) and EP (right) for the first biased data set (DS4) versus operating condition pressure for MPIC and UCLA experiment results.

\section{B. Biased test cell}

Biased test cell data were provided in two sets, DS4 and DS5, and the bias voltages of -10, 0, 10, and 20 Volts are chosen here for study to represent the wide range of positive and negative voltages. MPICs Poisson equation solver is required for analysis of biased test cell data in order to observe the effect of the new potential boundary conditions. Current collection for MPIC and UCLA experimental data can be seen in Figures 5-6 for DS4 and Figures 7-8 for DS5.
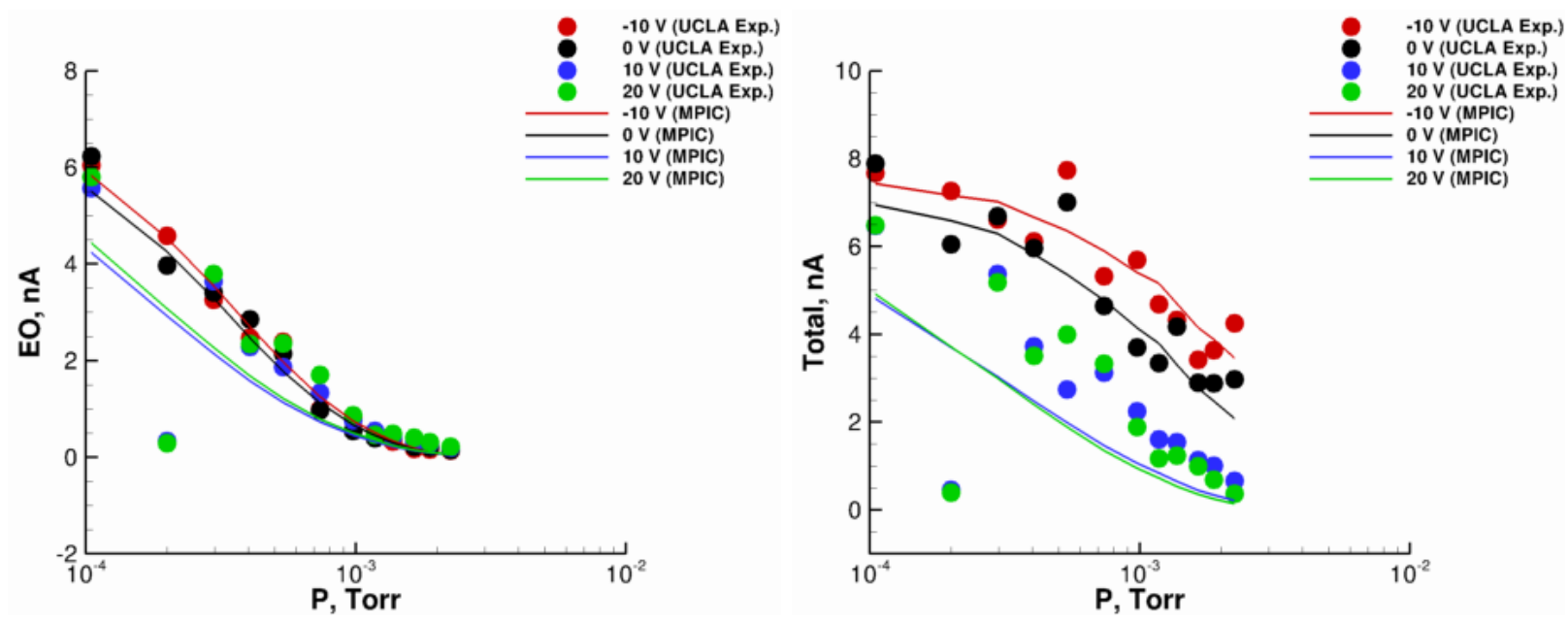

Figure 6. Current collection at the EO (left) and total domain (right) for the first biased data set (DS4) versus operating condition pressure for MPIC and UCLA experiment results.

DS4 data was collected at lower currents and with a smaller range of pressure operating conditions than the unbiased set. In Figure 5, the MPIC IC current collection matches well for the -10 and $0 \mathrm{~V}$ cases. The 10 and $20 \mathrm{~V}$ cases show good correlation for the lower-pressure region and then lie above the measurements for the higher-pressure region. The MPIC EP collection, however, diverges for most of the cases with the $-10 \mathrm{~V}$ case lying only slightly above the measurements. For DS4 there is more indication that there is unresolved beam divergence in the simulated ion beam resulting in off-axis ions being collected at the EP in the measurements.

For all DS4 currents, the agreement between MPIC and the measurements is the best for the $-10 \mathrm{~V}$ 

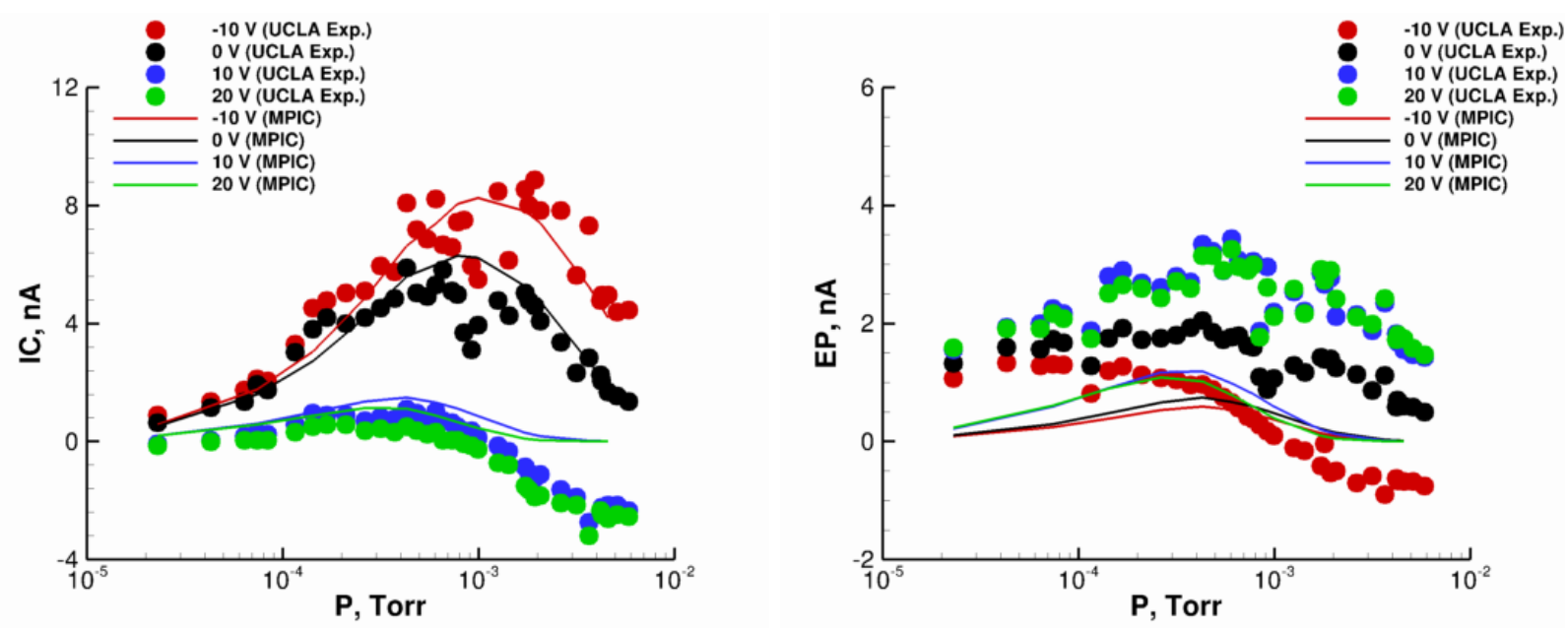

Figure 7. Current collection at the IC (left) and EP (right) for the second biased data set (DS5) versus operating condition pressure for MPIC and UCLA experiment results.

case. This ability of MPIC to correctly resolve the negatively biased case is consistent with the ion-only environment in which any secondarily produced stray electrons would be repelled electrostatically by the current collecting surface. Conversely, the positively-biased cases would be electron-collecting environments, an aspect not included in these simulations. Figure 6 shows the EO and total current, showing MPICs good correlation of currents.
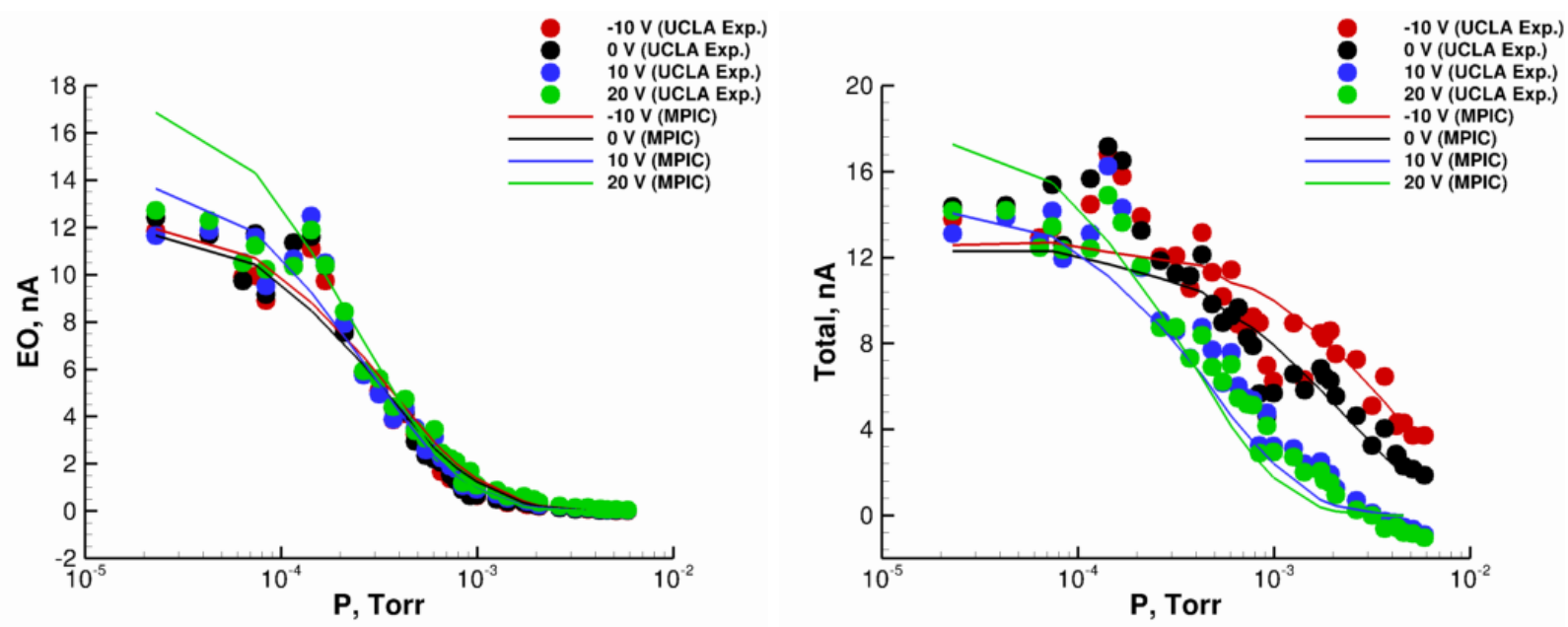

Figure 8. Current collection at the EO (left) and total domain (right) for the second biased data set (DS5) versus operating condition pressure for MPIC and UCLA experiment results.

DS5 data was collected at higher currents and with a larger range of pressure operating conditions. In Figure 7, again the MPIC IC current collection matches well with the measurements for the -10 and $0 \mathrm{~V}$ case. However, the MPIC EP collection lies mostly below the measurements, as seen in DS1 and DS5, with the $-10 \mathrm{~V}$ case being the closest match. The lower-pressure region again indicates an unresolved beam divergence in which $\sim 1 \mathrm{nA}$ of current is possibly being collected by the EP by off-axis ion. Additionally, DS5 further reinforces the idea that the negatively-biased cases are repelling any electrons in the environment and mimicking an ion-only operation, the reason for the very good correlation at all pressures of MPICs iononly simulation. Figure 8 shows the current at the EO and total current, showing MPIC's good correlation particularly for the -10 and $0 \mathrm{~V}$ cases.

An important aspect of the facility tests that has not been characterized experimentally is the divergence 

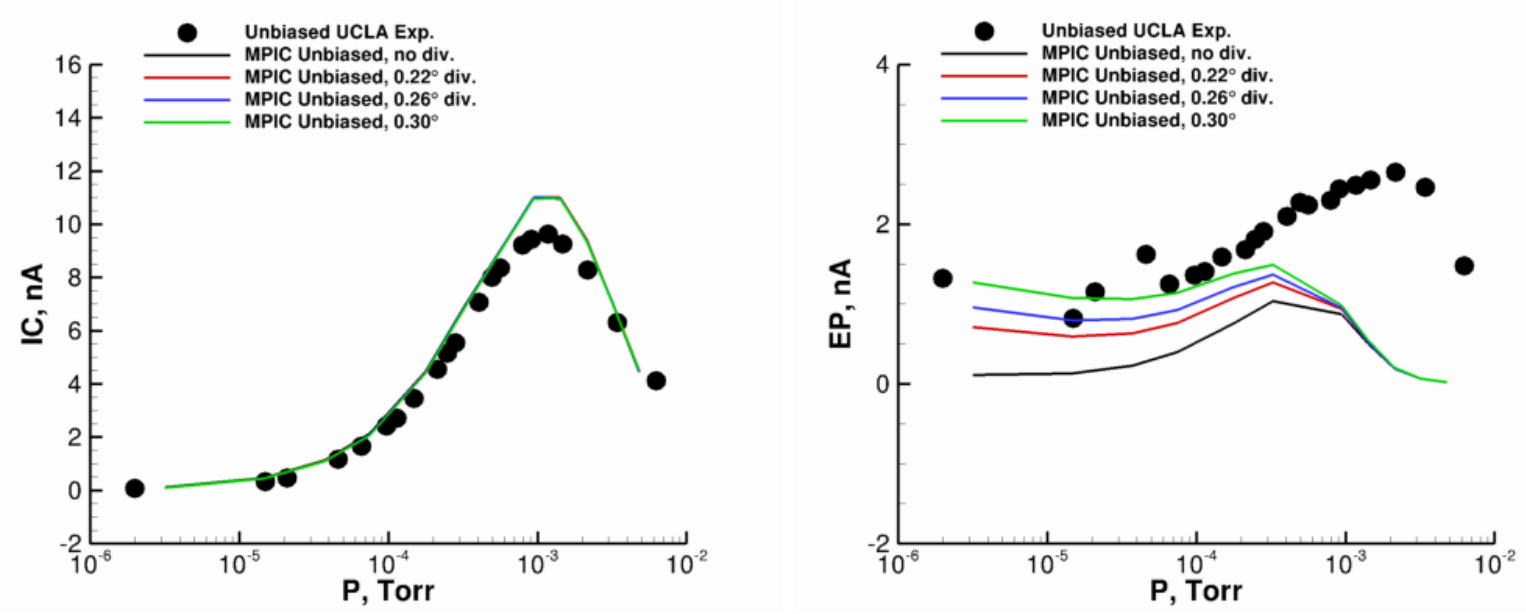

Figure 9. Current collection at the IC (left) and EP (right) for the unbiased data set (DS1) versus operating condition pressure illustrating MPIC sensitivity to beam divergence.

of the ion beam. Due to electrostatic repulsion, a small but finite level of beam divergence is to be expected. A series of numerical experiments is therefore conducted for the DS1 data set to assess any sensitivity on the computed results. Figure 9 shows the results of varying the beam divergence of the ion beam to angles of $0.22^{\circ}, 0.26^{\circ}$, and $0.30^{\circ}$. This set of beam divergences shows almost no change in the IC current collection and positive change in the EP current collection in the lower-pressure region. The estimated experimental beam divergence is approximately $0.30^{\circ}$, the closest matching curve. However, the variation of beam divergence does nothing to affect the higher-pressure current collection at the EP as all current collection collapses on a single trend. Both the Boltzmann relation and Poisson solver results show the same current collection behavior.

\section{Discussion}

One of the key observations from the comparisons between experimental data sets and ion-only simulations is the possible influence of electron physics at higher-pressure operating conditions. Most notably, the biased test cell data of DS4 and DS5 displays how well ion-only simulations perform when electrons are essentially forced out of current collection due to negative bias within the domain.

Given the reasonably controlled nature of the test cell facility, there are two ways in which electrons could be produced secondarily: secondary emission from the walls and secondary production via atomatom ionization. It is therefore useful to gauge the importance of the latter of these processes in the test cell environment. An analysis is carried out to determine the physical and numerical relevance of several "interactions of interest" in the test cell environment.

\section{A. Electron production via atom-atom and atom-ion interactions}

Atom-atom ionization is the multi-step process of energetic atoms interacting with other energetic atoms to create populations of excited atoms, ions, and electrons. This process is of interest to fundamental electric propulsion simulation as it may be an important source of energetic heavy species and electrons in addition to particle-induced secondary electron emission from a dielectric wall.

Atom-atom ionization and the presence of metastable states have been studied in the context of electric propulsion as well as other similar plasma and high-enthalpy environments. ${ }^{5,6}$ These particular interactions are difficult to quantify due to their generally smaller cross-sections as compared to atom-electron and ion-electron interactions. However, these interactions cannot be ignored for systems where atom-atom interactions could become a dominant process of the exchange of energy, charge, and the creation of secondary electrons.

The process of atom-atom ionization in the present study is described as follows. As fast ions are injected 
into the test cell at $\sim 46,900 \mathrm{~m} / \mathrm{s}$, momentum exchange (MEX) collisions are the dominant interaction process while symmetric charge exchange (CEX) collisions create populations of slow ions and fast atoms, an interaction which has recently been more fully described in MPIC. ${ }^{7}$ These fast atoms may interact with other slow atoms to create a population of electronically excited atoms. The $5 s^{2} 5 p^{5} 6 s^{1}$ configuration of xenon is chosen because it is the lowest electronically excited state and most easily accessible. ${ }^{8}$ Additional fast atoms further interact with this excited population to ionize them, creating a population of ions and secondary electrons. The process is described in Equations 4 through 6 .

$$
\begin{array}{rll}
\mathrm{Xe}_{\text {slow }}+\mathrm{Xe}_{\text {fast }}^{+} & \stackrel{\mathrm{CEX}}{\longrightarrow} \mathrm{Xe}_{\text {slow }}^{+}+\mathrm{Xe}_{\text {fast }} \\
\mathrm{Xe}_{\text {fast }}+\mathrm{Xe} & \stackrel{\text { excit. }}{\longrightarrow} \mathrm{Xe}^{*}+\mathrm{Xe} \\
\mathrm{Xe}_{\text {fast }}+\mathrm{Xe}^{*} \stackrel{\text { ioniz. }}{\longrightarrow} \mathrm{Xe}^{+}+\mathrm{Xe}+\mathrm{e}^{-}
\end{array}
$$

To calculate the relevance of atom-atom ionization within the xenon environment of the test cell, preliminary calculations are made for both atom-atom excitation and atom-atom ionization cross-sections along with associated mean free paths for densities relevant to the experiments. Due to the multi-step nature of this interaction, it is important to characterize both steps independently.

Firstly, the cross-section of the excitation of a xenon atom to the excited state via collisions with other xenon atoms are calculated. The cross-sections are calculated using Drawin's formula, ${ }^{9}$

$$
\begin{aligned}
\sigma_{\text {excit }}=4 \pi a_{0}^{2}\left(\frac{E_{1}^{H}}{E_{\text {excit }}}\right)^{2} \frac{m_{X e}}{m_{H}} & \xi_{0}^{2} f_{\text {excit }} \frac{2 m_{e}}{m_{e}+m_{X e}} \times \\
& \left(W_{\text {excit }}-1\right)\left[1+\frac{2 m_{e}}{m_{e}+m_{X e}}\left(W_{\text {excit }}-1\right)\right]^{-2}, \mathrm{~m}^{2}
\end{aligned}
$$

with $W_{\text {excit }}=E / E_{\text {excit }}, E$ being the free kinetic energy available for inelastic collisions, $E_{\text {excit }}$ is the excitation energy of the xenon metastable state, $f_{\text {excit }}$ is the absorption strength from ground to excited level, $\xi_{0}$ is the number of electrons in the outer (ground state) shell, $E_{1}^{H}$ is the first ionization energy level for hydrogen, $a_{0}$ is the Bohr radius, and $m_{e}$ and $m_{X e}$ are the masses of an electron and xenon, respectively.

The cross-section for the ionization of the xenon metastable state to singly-charged xenon ions are then calculated using Drawin's formula,

$$
\begin{aligned}
\sigma_{\text {ioniz }}=4 \pi a_{0}^{2}\left(\frac{E_{1}^{H}}{E_{\text {ioniz }}}\right)^{2} \quad \frac{m_{X e}}{m_{H}} \quad & \xi_{0}^{2} \frac{2 m_{e}}{m_{e}+m_{X e}} \times \\
& \left(W_{\text {ioniz }}-1\right)\left[1+\frac{2 m_{e}}{m_{e}+m_{X e}}\left(W_{\text {ioniz }}-1\right)\right]^{-2}, \mathrm{~m}^{2}
\end{aligned}
$$

with $W_{\text {ioniz }}=E / E_{\text {ioniz }}, E_{\text {ioniz }}$ is the ionization energy of xenon. Values for all parameters can be found in Table 1 .

\begin{tabular}{|c|c|}
\hline \hline Parameter & Value \\
\hline$E_{\text {excit }}$ & $9.03 \mathrm{eV}$ \\
\hline$E_{\text {ioniz }}$ & $12.13 \mathrm{eV}$ \\
\hline$E_{1}^{H}$ & $13.58 \mathrm{eV}$ \\
\hline$f_{\text {excit }}$ & 0.35 \\
\hline$\xi_{0}$ & 6 \\
\hline \hline
\end{tabular}

Table 1. Parameters used in the Drawin cross-section formulas for excitation and ionization.

Varying the available inelastic collision energy, $E$, from 100 to $2000 \mathrm{eV}$ in order to mimic the range of energy values within the accelerated beam of the test cell, Figure 10 shows a comparison of the Drawin cross-sections to the well-known atom-ion MEX/CEX collision cross-sections of Miller, et al., as well as collisions of atoms calculated using the variable hard-sphere (VHS) model. ${ }^{10,11}$ 


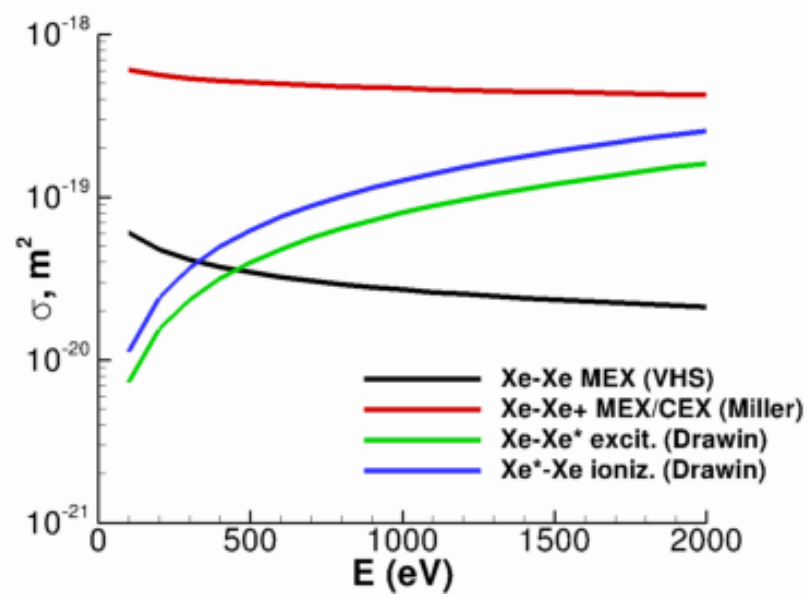

Figure 10. Comparison of elastic, MEX/CEX, excitation, and ionization cross-sections at relevant interaction energies.

Figure 10 shows that for relevant interaction energies the atom-atom excitation cross-section ranges from $4 \%$ of that of the MEX/CEX cross-section at $300 \mathrm{eV}$ to $27 \%$ at $1500 \mathrm{eV}$ and the atom-atom ionization crosssection ranges from $7 \%$ of that of the MEX/CEX cross-section at $300 \mathrm{eV}$ to $43 \%$ at $1500 \mathrm{eV}$, meaning that for much of this range the Drawin cross-sections are of the same order-of-magnitude as the main atom-ion cross-sections implying that these processes could be relevant in these environments. The VHS cross-sections are included in this comparison to illustrate the importance of simple, atom-atom collisions at lower energy levels with the other atom-atom interactions gaining dominance at the higher energy levels. The importance of these lower-probability atom-atom excitation and ionization can be argued to be statistically relevant due to the fact that there would be $\sim 0$ current collected at the IC without CEX.

One major drawback of this method of using the Drawin cross-sections, however, is that depending on the application, large uncertainties have been associated with their values. ${ }^{12}$ While studies have reported that actual cross-sections for atom-atom excitation and ionization can vary by an order of magnitude, the Drawin equations for cross-section are the only theoretical description of this type of atom-atom interaction. ${ }^{13}$

\section{B. Atom-atom cross-section analysis}

Next, an analysis is carried out in order to compare the mean free path, $\lambda$, of these atom-atom interactions at various relevant operating conditions to the radius of the test cell. A simple kinetic analysis is used to calculate the collision frequency, $\nu$, and mean free path for a given species number density,

$$
\begin{aligned}
c^{\prime} & =\sqrt{\frac{8 k_{B} T_{i}}{\pi m_{X e}}} \\
\nu & =n_{n} \sigma(E) g(E) \\
\lambda & =\nu / c^{\prime}
\end{aligned}
$$

with $c^{\prime}$ as the mean thermal speed of the species, $k_{B}$ as the Boltzmann gas constant, $T_{i}=298 \mathrm{~K}$ as the relatively cold temperature of all of the species, $m_{X e}$ as the mass of xenon, $\sigma(E)$ as the cross-section of interest, $g(E)$ as the relative velocity of the interaction which is calculated from conservation of momentum of electrostatics as $\sqrt{\frac{2 q \phi}{m_{X e}}}$ (thus both $\sigma$ and $g$ are dependant on the acceleration energy of the beam, $E$ ), and $n_{n}$ as the background species number density.

Figure 11 displays values for the atom-atom excitation mean free path, $\lambda$, for varying $n_{n}$ with parametric solutions for different interaction energies, $E$, compared to the radius of the test cell, $24.13 \mathrm{~mm}$. Each symbol on the curve represents a separate operating condition, or "run", of the test cell for DS1. Operating conditions which fall below the dashed line are conditions with mean free paths which are smaller than the test cell radius and therefore can be considered operating conditions for which the interaction has a 

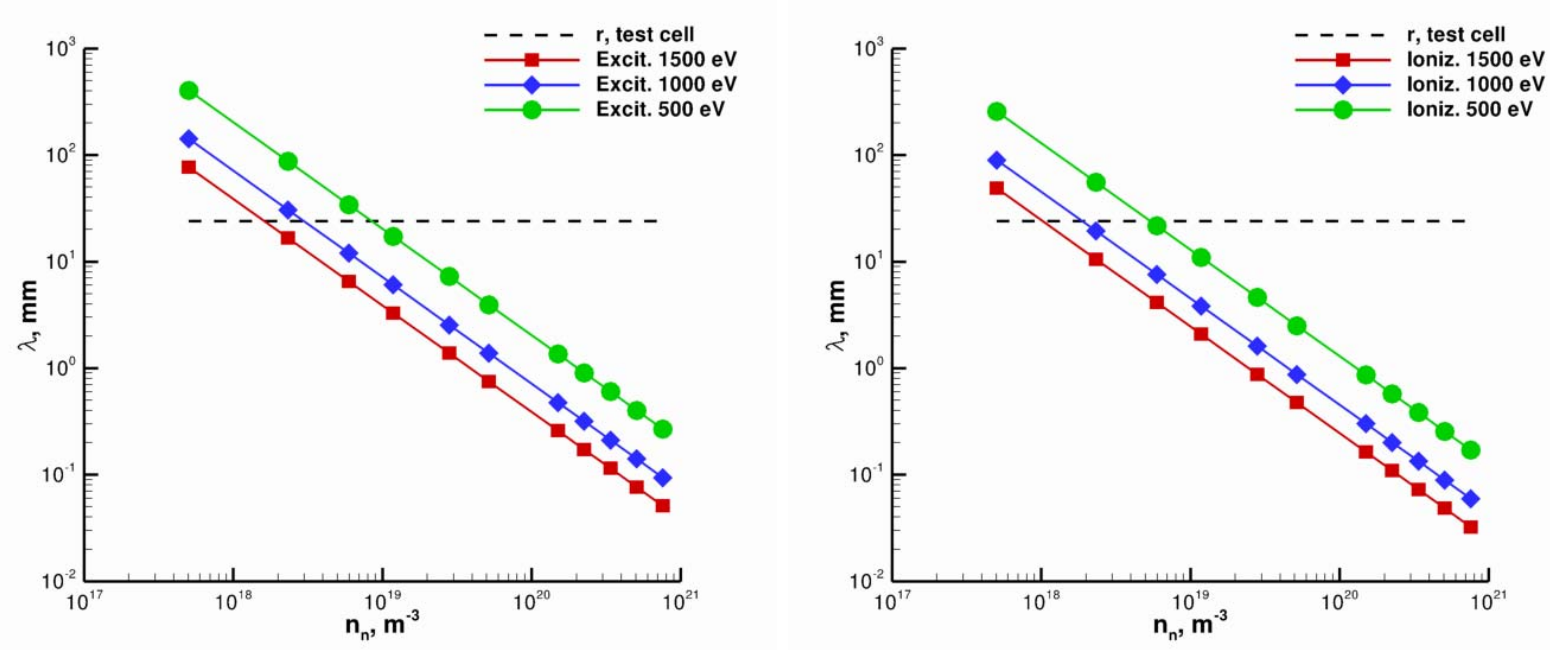

Figure 11. Comparison of the excitation (left) and ionization (right) mean free paths compared to the radius of the UCLA test cell as a function of possible interaction energies.

reasonable probability of occurring. Additionally, as the interaction energy decreases, the entire curve shifts upwards, resulting in less frequent interactions.

Figure 11 also displays values for the atom-atom ionization mean free path, $\lambda$, for varying $n_{n}$ and different interaction energies, $E$, compared to the radius of the test cell, $24.13 \mathrm{~mm}$. Operating conditions which fall below the dashed line are conditions with a more reasonable probability of ionization occurring. The reason for showing three decreasing values of interaction energy is to study the effects of either reducing the accelerating voltage as well as to show the effect on mean free path for colliding particles that might lose kinetic energy from collision to collision. A study could be carried out to attempt to quantify this second effect.

Based on these initial comparisons of mean free path to test cell geometry, atom-atom interactions should be likely to occur in our current operating conditions. Furthermore, for higher-pressure operating conditions, these interactions have mean free paths on the other of $1 / 100$ of the test cell radius, allowing for conditions in which many interactions could occur within the domain.

\section{Conclusion}

A wide array of comparisons between results obtained with the computational tool MPIC and experimental data from UCLA have been completed. A more physics-based electric potential solver was implemented into MPIC in order to allow for the simulation of various sets of biased and unbiased operating conditions. After reproducing current collection within the UCLA test cell at various ion beam currents and pressure regimes, a good characterization of the physics within the test cell has been established. In addition, the observation of a divergence between simulation and experiment under some conditions led to an analysis of the secondary production of electrons via atom-atom and atom-ion interactions. It was found that this type of secondary electron creation could be observed at the operating conditions in which poor agreement between simulation and experiment occurs.

As this study marks the completion of comparisons between various particle methods and experimental data, future work will focus on the inclusion of electron physics in the models used to simulated such physical environments. 


\section{Appendix}

The following tables describe the details of the total beam current per data set as well as the curve-fits used to create these currents as matched to the specific operating pressures of the experimental data.

Table 2. A table of the total current values used in MPIC for DS1 (left) and DS4 (right) derived from the experimental data through the curve-fits of Table 3

\begin{tabular}{|c|c|c|c|c|c|c|}
\hline & & DS4, $V_{\text {bias }}=$ & $-10 \mathrm{~V}$ & $0 \mathrm{~V}$ & $10 \mathrm{~V}$ & $20 \mathrm{~V}$ \\
\hline D.DI & $I$ & $\mathrm{P}$, Torr & $I_{i n}, \mathrm{nA}$ & $I_{i n}, \mathrm{nA}$ & $I_{i n}, \mathrm{nA}$ & $I_{i n}, \mathrm{nA}$ \\
\hline 1, 101T & & $1.05 \mathrm{E}-04$ & 7.33 & 6.92 & 5.12 & 5.29 \\
\hline $3.17 \mathrm{E}-06$ & $\begin{array}{l}16.73 \\
1670\end{array}$ & $2.00 \mathrm{E}-04$ & 7.08 & 6.61 & 4.22 & 4.31 \\
\hline $1.47 \mathrm{E}-05$ & 16.70 & $2.98 \mathrm{E}-04$ & 6.83 & 6.30 & 3.67 & 3.71 \\
\hline $3.75 \mathrm{E}-05$ & 16.63 & $4.06 \mathrm{E}-04$ & 6.57 & 5.98 & 3.24 & 3.24 \\
\hline 7.43E-05 & 16.52 & $5.37 \mathrm{E}-04$ & 6.27 & 5.61 & 2.85 & 2.81 \\
\hline $1.77 \mathrm{E}-04$ & 16.23 & 7.39E-04 & 5.82 & 5.09 & 2.41 & 2.33 \\
\hline $3.26 \mathrm{E}-04$ & 15.81 & $9.79 \mathrm{E}-04$ & 5.34 & 4.53 & 2.02 & 1.90 \\
\hline $9.45 \mathrm{E}-04$ & 14.19 & $1.18 \mathrm{E}-03$ & 4.97 & 4.11 & 1.76 & 1.62 \\
\hline $1.42 \mathrm{E}-03$ & 13.07 & $1.37 \mathrm{E}-03$ & 4.63 & 3.74 & 1.55 & 1.39 \\
\hline $2.13 \mathrm{E}-03$ & 11.55 & $1.65 \mathrm{E}-03$ & 4.19 & 3.27 & 1.29 & 1.11 \\
\hline $3.19 \mathrm{E}-03$ & 9.59 & $1.88 \mathrm{E}-03$ & 3.85 & 2.92 & 1.11 & 0.91 \\
\hline $4.78 \mathrm{E}-03$ & 7.26 & $2.25 \mathrm{E}-03$ & 3.37 & 2.44 & 0.86 & 0.64 \\
\hline
\end{tabular}

Table 3. A table of the total current values used in MPIC for DS5 (left) and equations for the curve-fits (right) used to calculate these numerical currents.

\begin{tabular}{|c|c|c|c|c|c|c|}
\hline $\mathrm{DS} 5, V_{\text {bias }}=$ & $-10 \mathrm{~V}$ & $0 \mathrm{~V}$ & $10 \mathrm{~V}$ & $20 \mathrm{~V}$ & & \\
\hline $\mathrm{P}$, Torr & $I_{\text {in }}, \mathrm{nA}$ & $I_{\text {in }}, \mathrm{nA}$ & $I_{\text {in }}, \mathrm{nA}$ & $I_{\text {in }}, \mathrm{nA}$ & & \\
\hline $2.30 \mathrm{E}-05$ & 12.44 & 12.19 & 14.13 & 17.39 & DS1 & $I_{\text {in }}=16.74 \exp (-174.7 P)$ \\
\hline $7.40 \mathrm{E}-05$ & 12.29 & 11.97 & 13.25 & 15.88 & DS4 $-10 \mathrm{~V}$ & $I_{i n}=7.61 \exp (-362.42 P)$ \\
\hline $1.43 \mathrm{E}-04$ & 12.09 & 11.68 & 12.15 & 14.04 & $0 \mathrm{~V}$ & $I_{i n}=7.28 \exp (-485.84 P)$ \\
\hline 2.63E-04 & 11.75 & 11.19 & 10.44 & 11.34 & $10 \mathrm{~V}$ & $I_{i n}=-1.39 \ln (P)-7.61$ \\
\hline 4.31E-04 & 11.28 & 10.54 & 8.45 & 8.41 & $20 \mathrm{~V}$ & $I_{i n}=-1.52 \ln (P)-8.62$ \\
\hline $6.06 \mathrm{E}-04$ & 10.82 & 9.90 & 6.78 & 6.16 & DS5 $-10 \mathrm{~V}$ & $I_{i n}=12.51 \exp (-239.32 P)$ \\
\hline 7.81E-04 & 10.38 & 9.30 & 5.43 & 4.51 & $0 \mathrm{~V}$ & $I_{i n}=12.30 \exp (-358.05 P)$ \\
\hline $9.96 \mathrm{E}-04$ & 9.86 & 8.61 & 4.14 & 3.07 & $10 \mathrm{~V}$ & $I_{i n}=14.55 \exp (-1260.73 P)$ \\
\hline $1.73 \mathrm{E}-03$ & 8.27 & 6.62 & 1.64 & 0.83 & $20 \mathrm{~V}$ & $I_{i n}=18.12 \exp (-1780.76 P)$ \\
\hline $2.07 \mathrm{E}-03$ & 7.61 & 5.85 & 1.06 & 0.45 & & \\
\hline $3.66 \mathrm{E}-03$ & 5.20 & 3.31 & 0.14 & 0.03 & & \\
\hline 4.60E-03 & 4.16 & 2.37 & 0.04 & 0.01 & & \\
\hline
\end{tabular}

\section{Acknowledgments}

The authors would like to thank Lauren Chu, Marlene Patino, and Dr. Richard Wirz of UCLA, Dr. Nate Lockwood at Kirtland AFB, Dr. Justin Koo at Edwards AFB, ERDC DSRC, and Dr. Chunpei Cai at New Mexico State University. This work was supported by the University of Michigan/AFRL Center of Excellence in Electric Propulsion (MACEEP), Grant \#F9550-09-1-0695. 


\section{References}

${ }^{1}$ R. E. Wirz, L. Chu, M. Patino, H. Mao, and S. Araki. Well-Characterized Plasma Experiments for Validation of Computational Models, IEPC-2011-122. In 32nd International Electric Propulsion Conference, Wiesbaden, Germany, 2011.

${ }^{2}$ Y. Choi. Modeling an anode layer Hall thruster and its plume. Doctoral dissertation, University of Michigan, 2008.

${ }^{3}$ I.D. Boyd and J.T. Yim. Modeling of the near field plume of a Hall thruster. Journal of applied physics, 95(9):4575, 2004.

${ }^{4} \mathrm{C}$ Cai. Theoretical and numerical studies of plume flows in vacuum chambers. PhD thesis, University of Michigan, 2005.

${ }^{5}$ R. Dressler, Y. Chiu, O. Zatsarinny, K. Bartschat, R. Srivastava, and L. Sharma. Near-infrared collisional radiative model for Xe plasma electrostatic thrusters: the role of metastable atoms. Journal of Physics D: Applied Physics, 42(18):185203, September 2009.

${ }^{6}$ A. Bogaerts and R. Gijbels. The role of fast argon ions and atoms in the ionization of argon in a direct-current glow discharge: A mathematical simulation. Journal of Applied Physics, 78(11):6427, 1995.

${ }^{7}$ P. N. Giuliano and I. D. Boyd. Effects of detailed charge exchange interactions in DSMC-PIC simulation of a simplified plasma test cell. In 32nd International Electric Propulsion Conference, Wiesbaden, Germany, 2011.

${ }^{8}$ K. E. Harwell and R. G. Jahn. Initial Ionization Rates in Shock-Heated Argon, Krypton, and Xenon. Physics of Fluids, $7(2): 214-222,1964$.

${ }^{9}$ H. W. Drawin and F. Emard. Atom-atom excitation and ionization in shock waves of the noble gases. Physics Letters, 43(4):333-335, 1973.

${ }^{10}$ J. S. Miller, S. H. Pullins, D. J. Levandier, Y. Chiu, and R. A. Dressler. Xenon charge exchange cross sections for electrostatic thruster models. Journal of Applied Physics, 91(3):984-991, 2002.

${ }^{11} \mathrm{G}$. A. Bird. Molecular gas dynamics and the direct simulation of gas flows. Clarendon, 1994.

${ }^{12}$ H. H. Fleischmann and R. C. Dehmel. On Drawin's formula for ionization in atom-atom collisions. Zeitschrift fr Physik, 252(5):435-442, October 1972.

${ }^{13} \mathrm{M}$. G. Kapper. A high-order transport scheme for collisional-radiative and nonequilibrium plasma. PhD thesis, The Ohio State University, 2009. 